\title{
Sizing Nanomatter in Biological Fluids by Fluorescence Single Particle Tracking
}

\author{
Kevin Braeckmans, ${ }^{\dagger, \uparrow}$ Kevin Buyens, ${ }^{\dagger, \uparrow}$ Wim Bouquet, ${ }^{\ddagger}$ Chris Vervaet, ${ }^{\ddagger}$ Philippe Joye, ${ }^{\S}$

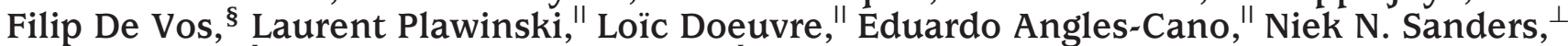 \\ Jo Demeester, ${ }^{\dagger}$ and Stefaan C. De Smedt* ${ }^{\dagger}$
}

${ }^{\dagger}$ Laboratory of General Biochemistry and Physical Pharmacy, ${ }^{\ddagger}$ Laboratory of Pharmaceutical Technology, and

${ }^{\S}$ Laboratory of Radiopharmacy, Ghent University, Harelbekestraat 72, 9000 Gent, Belgium, "Inserm U919,

UMR CNRS 6232, GIP Cyceron, Bd Henri Becquerel, 14074-cedex, Caen, France, and ${ }^{\perp}$ Laboratory of Gene Therapy,

Ghent University, Heidestraat 19, 9820 Merelbeke, Belgium

\begin{abstract}
Accurate sizing of nanoparticles in biological media is important for drug delivery and biomedical imaging applications since size directly influences the nanoparticle processing and nanotoxicity in vivo. Using fluorescence single particle tracking we have succeeded for the first time in following the aggregation of drug delivery nanoparticles in real time in undiluted whole blood. We demonstrate that, by using a suitable surface functionalization, nanoparticle aggregation in the blood circulation is prevented to a large extent.
\end{abstract}

KEYWORDS Nanobiophotonics, nanoparticles, drug delivery, nanomedicines, medical diagnostics

T here is a growing appreciation that a thorough understanding of the fundamental interactions of nanoparticles with biological materials is required to make the transition from the laboratory to a successful and safe product. ${ }^{1}$ In particular, the interaction with plasma and blood components is currently of great interest since many types of nanoparticles are intended for intravenous administration. Examples are functionalized nanoparticles for biomedical imaging such as gold nanoparticles for X-ray computer tomography or quantum dots for fluorescence imaging. ${ }^{2}$ In the drug delivery field, nanomedicines are being developed based on liposomal or polymeric carrier materials to deliver therapeutic agents, such as anticancer drugs and nucleic acids, to specific target tissues. ${ }^{3,4}$ While nanoparticles can be accurately characterized in simple solvents at the time of preparation, their efficacy will ultimately be determined by their interaction with biopolymers and other constituents of the blood circulation. For instance, recent investigations have shown that typically a biopolymer corona is formed around nanoparticles in plasma, depending on their composition, size, and surface charge. ${ }^{5}$ Since this corona alters their outer surface, it can influence the effective nanoparticle behavior. ${ }^{6}$

Apart from their surface properties, a crucial parameter is the effective size of nanoparticles in the blood circulation because it directly influences their clearance and biodistribution, ${ }^{7-10}$ as well as their subsequent uptake and processing

\footnotetext{
* To whom correspondence should be addressed. Tel: +32 9264.80.76. Fax: +32 9264 0.81.89. E-mail: Stefaan.Desmedt@UGent.be.

ๆ These authors contributed equally to this work.

Received for review: 06/25/2010

Published on Web: 10/05/2010
}

by target cells. ${ }^{11,12}$ Furthermore, it is suggested that the effective size of nanoparticles might be related to their toxicity. ${ }^{13}$ Despite its importance, due to a lack of appropriate techniques no studies have been reported so far where the size and aggregation of nanoparticles has been studied directly in undiluted biological fluids. While dynamic light scattering (DLS) is arguably the most successful technique to size nanoparticles in simple solvents, it is incompatible with biological fluids since the particles of interest should be dispersed in a solution free of other light scattering components. Imaging techniques such as atomic force microscopy and electron microscopy suffer from a poor statistical power and require extensive preparation and fixation steps that could result in morphological deformation and misleading measurements. In a recent study, nanoparticle tracking and analysis (NTA) was explored for sizing gold nanoparticles in plasma. However, since the NTA technique relies on the detection of scattered light, plasma had to be diluted $10^{6}$ times for the nanoparticles to be visible. ${ }^{14}$ In another recent study, sizing of polystyrene model particles in plasma was achieved with success using differential centrifugal sedimentation although again the plasma was diluted at least 50 times. ${ }^{6}$ Flow cytometry was recently explored as an alternative method for sizing fluorescently labeled nanomedicines in serum. ${ }^{15}$ However, it was recognized that interpretation of absolute size values should be done with caution as the data quantification depends on the shape, surface structure, and refractive index of the particles.

Here we report on the first study on the effective size of nanoparticles in undiluted biological fluids like plasma and whole blood by using fluorescence single particle tracking (fSPT). fSPT is a microscopy technique that allows one to 


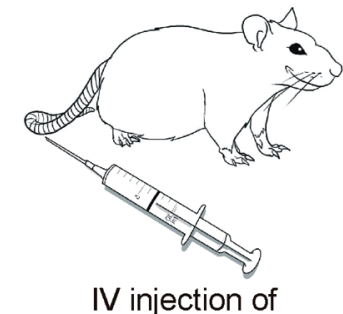

fluorescent nanoparticles

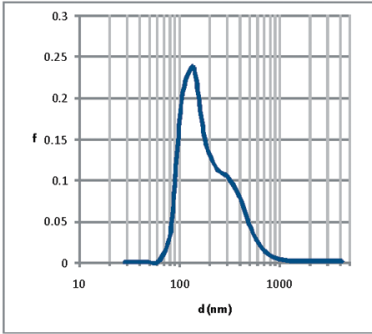

Nanoparticle size distribution (MEM analysis)

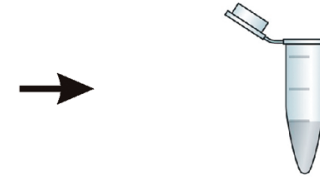

Blood sample collection

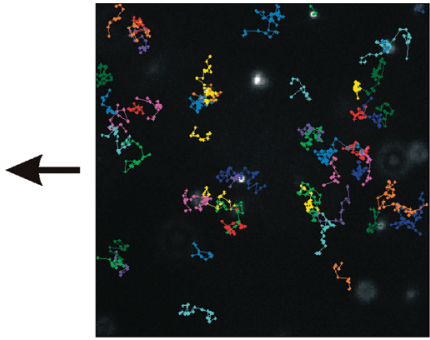

Calculate individual trajectories

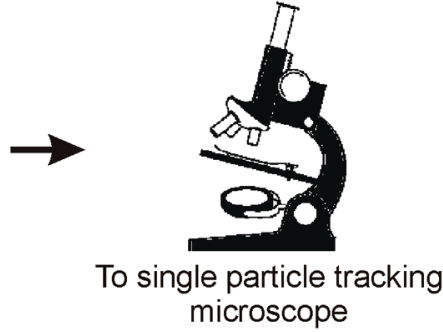

)

FIGURE 1. Schematic overview of fSPT sizing of drug nanoparticles in blood. The nanoparticle formulation is injected in either an artificial blood circulation system or in a lab animal (rat). Next, small blood samples are collected at regular time intervals and transferred to the fSPT microscope. After recording movies of the diffusing nanoparticles, their trajectories are calculated using image processing software. Finally, the diffusion coefficient is estimated for each individual particle, which is then converted to a continuous size distribution using MEM analysis.

image the movement of individual fluorescently labeled nanoparticles with nanometer precision and has mostly been used for studying the mobility of biomolecules and nanomedicines in living cells. ${ }^{16-19}$ While it has been previously realized that fSPT can be used for measuring the size of hard sphere colloids in a simple dispersion, ${ }^{20}$ here we demonstrate for the first time that this technique, when combined with maximum entropy analysis, allows one to measure in real-time accurate and precise size distributions of nanoparticles dispersed in undiluted biological fluids. After a detailed validation, we apply fSPT to study the timedependent aggregation of liposomes with different surface functionalizations in whole blood, both in vitro and in vivo after intravenous injection in rats. Finally, we demonstrate that fSPT is a promising diagnostics tool for detecting and characterizing blood-borne cell-derived microparticles.

As is illustrated in Figure 1, fSPT sizing is based on imaging the diffusional motion of fluorescently labeled particles using a highly sensitive fluorescence microscope setup (see Materials and Methods in Supporting Information). Because of the fluorescent labeling, the particles can be seen as bright dots on a dark background. Using custom developed software, the movies are analyzed to obtain the motion trajectory of each individual particle. By calculating the diffusion coefficient for each trajectory, a distribution of empirical diffusion coefficients can be obtained that can be transformed to a size distribution with the Stokes-Einstein equation. As discussed in the Theory section of the Supporting Information, the precision of the raw size measurement can be substantially refined by a maximum entropy decon- volution method (MEM) that eliminates sampling noise and statistical broadening of the distribution. The power and usefulness of adding MEM analysis to fSPT is demonstrated by computer simulations in the Supplementary Results of the Supporting Information. Futhermore, the fSPT sizing technique was successfully validated against standard DLS measurements using dispersions of 100 and $200 \mathrm{~nm}$ fluorescent nanospheres (see Supplementary Results in Supporting Information). While similar average sizes and modes were found using both techniques, the resolution of fSPT combined with MEM analysis is substantially better as compared to DLS. Furthermore, contrary to DLS, by fSPT it was very well possible to measure the size distribution of nanospheres in full human serum (see Supplementary Results and Figure S3 in Supporting Information).

Having established that fSPT allows one to measure accurate size distributions of nanoparticles in biological fluids, we subsequently investigated whether we can monitor the size of liposomes that have been under investigation for decades as nanocarriers for drugs in serum, plasma, and whole blood. Cationic liposomes were made out of equimolar amounts of the cationic lipid 1,2-dioleyl-3-trimethylammoniumpropane (DOTAP) and the neutral fusogenic lipid 1,2-dioleoyl-sn-glycero-3-phosphoethanolamine (DOPE). The DOTAP-DOPE liposomes were fluorescently labeled by incorporating $0.1 \mathrm{~mol} \%$ DOPE-LissamineRhodamineB. Blood was collected from three healthy donors, a part of which was used to prepare serum and plasma. The serum, plasma, or blood was transferred into an artificial blood circulation system, as illustrated in Supporting Information Figure S5. 
a

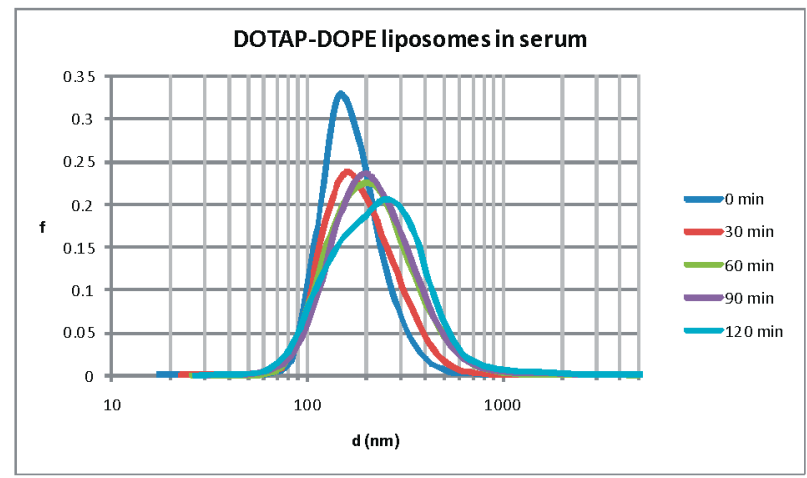

b

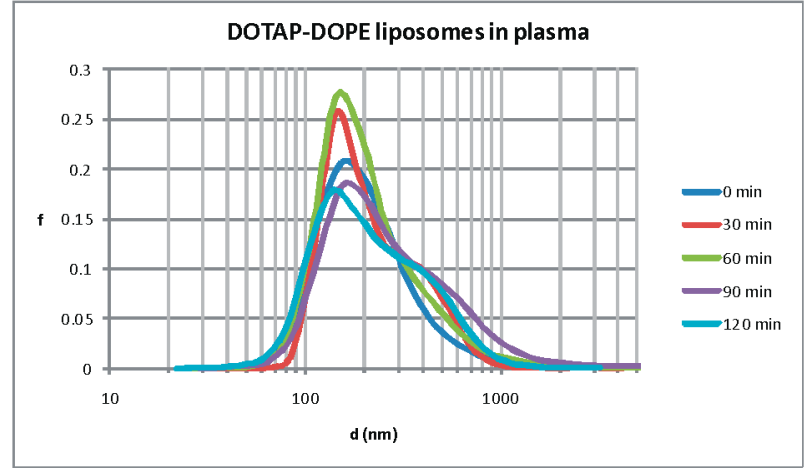

C

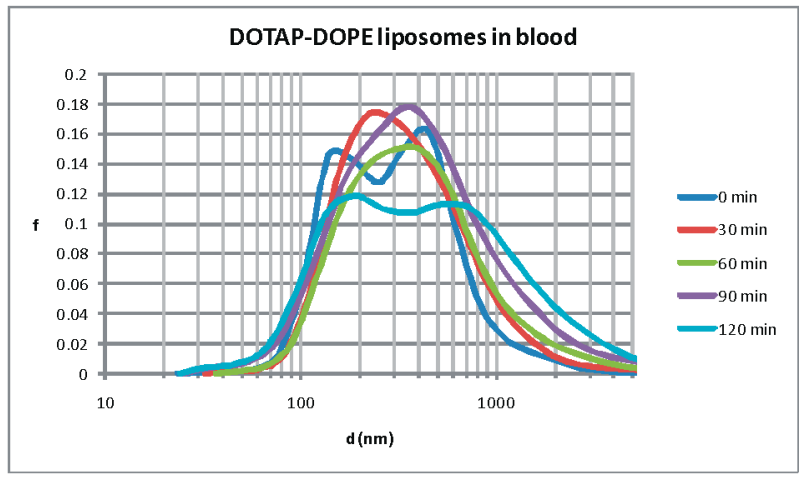

d

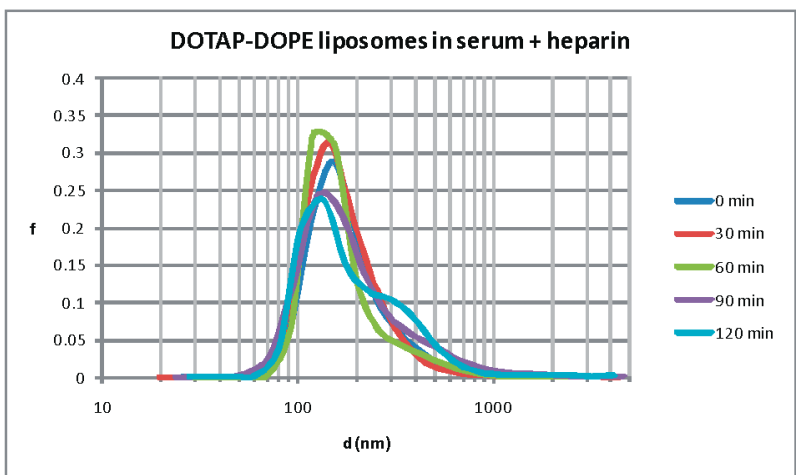

FIGURE 2. fSPT sizing of nonpegylated DOTAP-DOPE liposomes in serum, plasma, and whole blood. (a) Serum, (b) plasma, (c) whole blood, and (d) heparin-containing serum was transferred to the artificial blood circulation system. DOTAP-DOPE liposomes were injected and their size distribution was measured by fSPT at regular time intervals.

After equilibration of the circulation system to $37^{\circ} \mathrm{C}$, the DOTAP-DOPE liposomes were injected. Small samples were collected and measured by fSPT every 30 min over $2 \mathrm{~h}$. The size distributions are shown in Figure 2 and were obtained by pooling the data from three donors and applying MEM analysis. The size distributions are visualized as the relative frequency $\alpha_{i}$ with which a size $d_{i}$ is present in the particle population (also see the Theory section in the Supporting Information). For each size distribution, the sum of all frequencies equals $1: \sum \alpha_{i}=1$. The ordinate axis of the size distributions is labeled as " $f$ ", which is the more conventional notation for "frequency", rather than $\alpha$. In serum, a slight tendency toward larger aggregates is noticeable over time (Figure 2a). In plasma, the aggregation over time is slightly more pronounced (Figure $2 \mathrm{~b}$ ) but still is far less than what happens in whole blood (Figure 2c). Compared to serum and plasma, the aggregation in whole blood is proceeding much faster and leads to substantially larger aggregates of several micrometer in diameter. This can also clearly be seen in the videos provided as Supporting Information (Supplementary Movies 2 to 4). In plasma, a clear increase in particle intensity can be seen as compared to serum, which is a visual confirmation that liposomes are coagulating to form larger particles. In blood, one can even see micrometer-sized aggregates (see Supporting Information Supplementary Movie 4a) next to smaller particles (see Supporting Information Supplementary Movie $4 b$ ). These movies are a visual confirmation that the increase in size as measured by fSPT in
Figure 2 is effectively due to liposome aggregation and not for instance merely by binding of the original liposomes to larger blood components. Taken together, these results suggest that serum and even plasma are no reliable substitutes for whole blood when it comes to studying the size of nanoparticles after intravenous injection.

To ensure that the more severe aggregation of the cationic liposomes in plasma and blood was not induced by (anionic) heparin, which is used as an anticoagulant, we performed fSPT sizing experiments on serum samples containing Liheparin. This was done by transferring the serum into similar Li-heparin tubes as were used for collection of the blood samples. After an incubation period of $15 \mathrm{~min}$, the heparin containing serum was transferred to the artificial blood circulation and the liposomes were injected as before. The results are shown in Figure $2 \mathrm{~d}$. While the size distributions are not identical to the ones in Figure 2a, which is to be expected since aggregation is a random process, it can be seen that the presence of heparin does not increases the rate or extent of aggregation. While further research is needed, aggregation could come from the adsorption of soluble proteins, such as fibrinogen, which is present at a high concentration in plasma and blood but not in serum. Such proteins may adsorb to the surface of the charged DOTAP-DOPE liposomes and act as a bridge between them with the formation of larger aggregates.

Covering the surface of (drug loaded) nanoparticles with polyethylene-glycol (PEG) chains is currently the most prom- 
ising method to avoid aggregation in the bloodstream and subsequent clearance by the reticulo-endothelial system (RES). Pegylation also allows altering the biodistribution of the nanoparticles to achieve optimal pharmacokinetics. ${ }^{21,22}$ Here we have used the fSPT method to examine for the first time if pegylation indeed suppresses the aggregation of liposomes in undiluted whole blood. A first type of pegylated DOTAP-DOPE liposomes was prepared through the addition (10 mol \%) of 1,2-distearoyl-sn-glycero-3-phosphoethanolamine- $N$-[methoxy (polyethylene glycol)-2000] (DSPE-PEG). A second type of pegylated liposomes was obtained using $10 \mathrm{~mol} \% \mathrm{~N}$-palmitoyl-sphingosine-1-\{ succinyl[methoxy(polyethylene glycol)2000]\} (ceramide-PEG) containing a C16 acyl chain. It is believed that in contrast to DSPE-PEG ceramide-PEG lipids can slowly diffuse out of the liposome when injected in blood, thereby providing a form of sheddable PEG coat. This sheddable coat improves the drug release properties from endocytosed liposomes when compared to liposomes containing a conventional, nonsheddable PEG coat that is obtained by the inclusion of DSPE-PEG. ${ }^{23}$ After injection of the liposomes into the artificial blood circulation system, fSPT size measurements were performed over $2 \mathrm{~h}$ in 30 min time intervals. The experiment was repeated three times with blood from different donors. The trajectories from the three blood samples were pooled for each time point before performing the MEM size analysis. As is evident from Figure $3 \mathrm{a}$, inclusion of $10 \mathrm{~mol} \%$ of DSPE-PEG in DOTAPDOPE liposomes indeed prevented aggregation in blood when compared to the nonpegylated liposomes (compare Figures $3 \mathrm{a}$ and 2c). Nevertheless, aggregation still occurs, although much less, as can be clearly seen from the distributions at 90 and $120 \mathrm{~min}$. In addition, we tested the effect of including less DSPE-PEG (5 and $3 \mathrm{~mol} \%$ ), showing that aggregation becomes progressively worse as the pegylation degree decreases (see Supporting Information Figure S6). When compared to DSPE-PEG, ceramide-PEG liposomes (containing $10 \mathrm{~mol} \%$ of $\mathrm{C} 16$ ceramide-PEG) prove to be much more prone to aggregation (Figure $3 \mathrm{~b}$ ) although still less than the nonpegylated ones (Figure 2c). This can be explained by the ability of the ceramide-PEG molecules to gradually diffuse out of the liposomes. ${ }^{24}$ Indeed, the lipid exchangeability of the ceramide anchor of the ceramide-PEG conjugate allows its redistribution in other lipid bilayers that are abundantly present in blood, for example, the plasma membrane of blood cells. As recently studied by Akinc et al., small changes in the anchor chain length of PEGceramide analogues can result in significant effects on in vivo efficacy. ${ }^{25}$ Let it be noted that the size distributions measured in whole blood at the first time point in Figure 3a,b correspond very well to the size distributions of the liposomes measured in buffer, providing further evidence that fSPT indeed allows to measure correctly the size of liposomes in complex biological fluids.

An ultimate ambition of drug delivery scientists designing nanoparticles for intravenous administration is to get a clear a

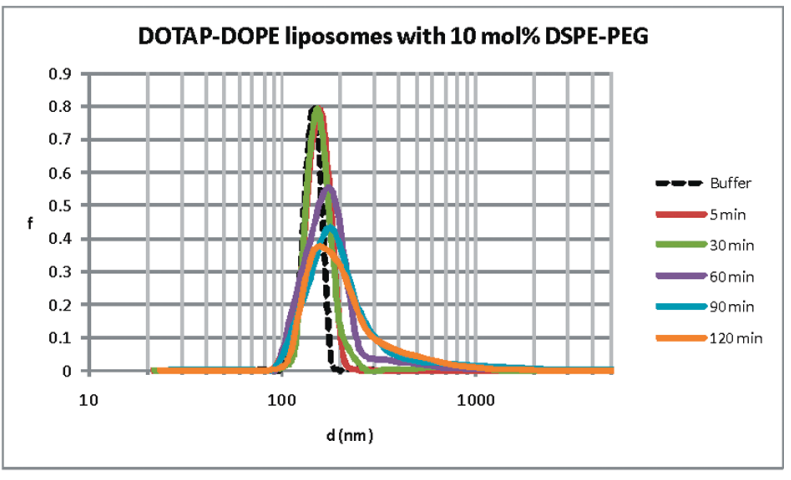

b

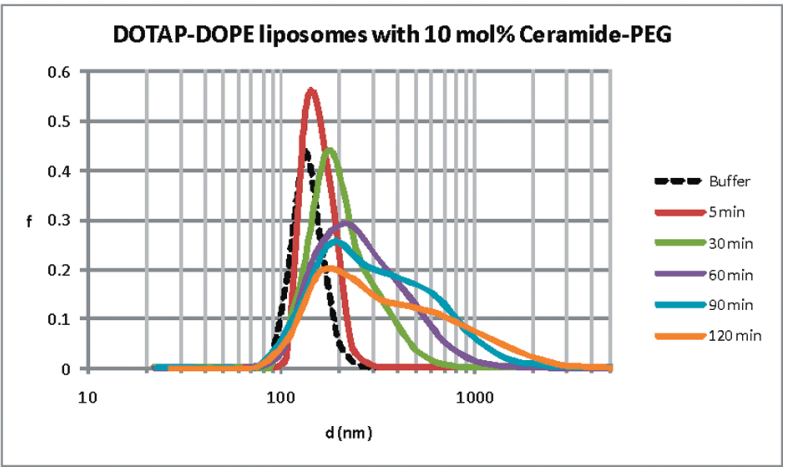

FIGURE 3. Pegylation of liposomes decreases their tendency to aggregate in whole blood. (a) fSPT sizing reveals that DOTAP-DOPE liposomes pegylated with $10 \mathrm{~mol} \%$ DSPE-PEG remain stable in whole blood over an extended period of time. (b) Using $10 \mathrm{~mol} \mathrm{\%}$ ceramide-PEG instead, the liposomes again aggregate over time due to the ability of ceramide-PEG to diffuse out of the liposomes.

view on the size and aggregation of nanoparticles after intravenous injection in lab animals or patients. As fSPT sizing in whole blood is feasible and only requires samples of a few microliters, we took the challenge to analyze by fSPT liposomes that were first IV injected in rats and then collected from the rats without euthanizing the lab animals. For each of the three types of liposomes, three rats were injected with $200 \mu$ L of liposome suspension after which small blood samples $(50-100 \mu \mathrm{L})$ were taken in Li-heparin vials at several time points after injection. Nonpegylated liposomes were found to be severely aggregated already after 5 min (Figure 4b). After 20 and $40 \mathrm{~min}$, however, we noticed that the larger particles had disappeared from the blood samples, resulting in size distributions that were shifted to smaller sizes. This observation confirms that larger particles are more rapidly removed from the bloodstream than smaller ones. After 60 min only, very few liposomes could still be detected due to which no meaningful size distribution could be calculated. For the $10 \mathrm{~mol} \%$ ceramide-PEG liposomes, we observed some aggregation at $5 \mathrm{~min}$ after injection (Figure 4c). Similar to the nonpegylated liposomes, at later times after injection no aggregates could be seen anymore while the number of particles in the fSPT movies was steadily decreasing. For the 10 mol \% DSPE-PEG liposomes (Figure $4 d$ ), the number of particles did not decrease so rapidly, though it is again clear from the distributions that the larger 
a

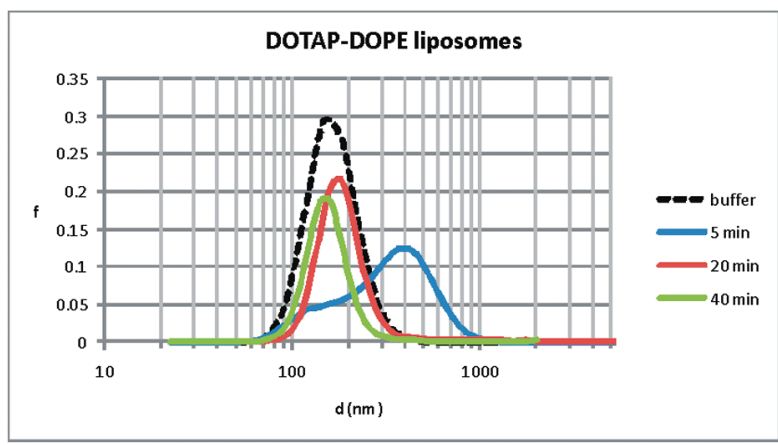

b

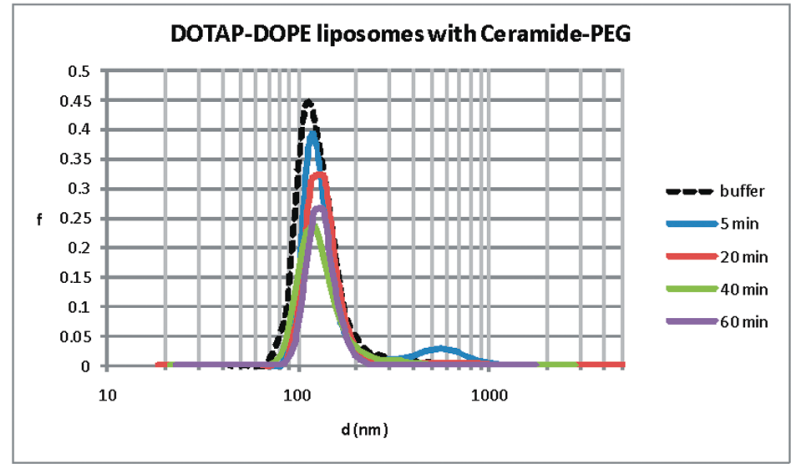

C

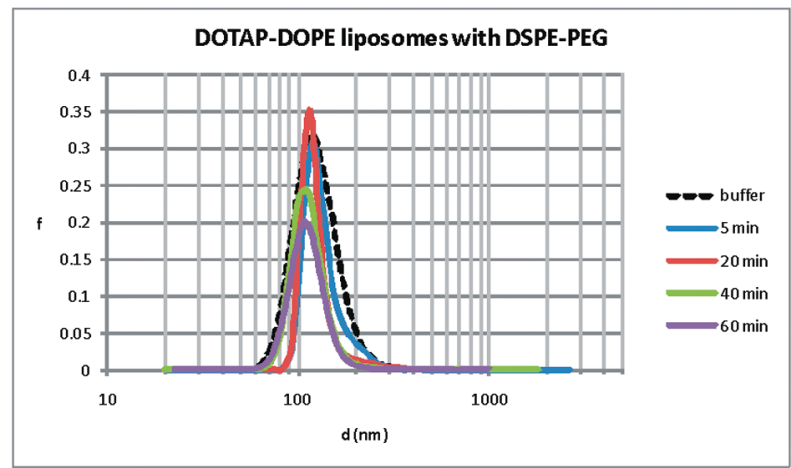

FIGURE 4. DOTAP-DOPE liposomes were intravenously injected in rats $(n=3)$ after which small blood samples were collected at regular time intervals for fSPT size measurements. (a) Nonpegylated liposomes immediately form large aggregates that are quickly removed from the blood circulation. (b) fSPT measurements on liposomes with $10 \mathrm{~mol} \%$ ceramide-PEG indicate a limited amount of aggregates at early time points which are removed at later times. (c) Liposomes with $10 \mathrm{~mol} \%$ DSPE-PEG remain stable over the entire measurement period.

particles are removed first. From these in vivo experiments, we conclude that the sizing of nanoparticles in blood should at first be performed in a closed artificial blood circulation system since aggregates are quickly removed from the bloodstream in vivo, which may lead to an underestimation of the extent of aggregation.

To demonstrate that fSPT sizing is not limited to the study of exogenous nanoparticles, we have used this technique for the detection, identification, and sizing of endogenous cellderived microparticles (MPs). Cell-derived MPs are particles in the 0.1 to $1 \mu \mathrm{m}$ range derived from the plasma membrane of activated cells or cells entering the apoptotic pathway. ${ }^{26}$ The characterization of MPs is of importance to the medical diagnostics field as they could be useful as biomarkers for metabolic and systemic diseases, as well as for thrombosis (myocardial infarction, stroke) and cancer. ${ }^{27,28}$ Apart from their detection, sizing of MPs is of particular interest as it provides for an additional confirmation that the detected particles are indeed cell-derived MPs and not exosomes $(<100 \mathrm{~nm})$ or apoptotic bodies $(>1 \mu \mathrm{m})$ coming from apoptotic cells. Furthermore, by using fSPT it is possible to address the currently open question of whether there is a relationship between the size of the MPs and their cellular origin. For example, it has been suggested that plateletderived MPs are around $200 \mathrm{~nm}$, whereas endothelialderived MPs could be around $400 \mathrm{~nm} .{ }^{29}$ The accurate sizing of cell-derived MPs is of additional interest to investigate whether their size is related to the type of stimulus or the disease background (inflammatory, cancer, etc.) ${ }^{30}$ As such, it is clear that the size of MPs is a potential important pathophysiological parameter. Cell-derived MPs typically expose phosphatidylserine at the outer leaflet of the membrane that is normally localized in the internal leaflet of normal quiescent cells. They also display identity antigens specific for the parent cell, as well as functional glycoproteins. By using specific fluorescently labeled antibodies directed against these glycoproteins or annexin V, which targets phosphatidylserine, not only size determination in plasma by fSPT should be possible, but also concomitant identification of their cellular origin and their functional characterization. To demonstrate the possibility of detecting and sizing cell-derived MPs in plasma, MPs were collected from human microvascular endothelial cells in culture. Next, Annexin V-FITC or antibodies against CD105 or UPA were added for labeling of the endothelial MPs. As a negative control, fluorescently labeled antibodies against CD 41 were added, which is a platelet molecular cluster of differentiation that is not present in endothelial MPs. Human microparticlefree undiluted plasma was spiked with endothelial MPs and examined by fSPT. As shown in the Supplementary Movies 5, 6 and 7 in Supporting Information, endothelial MPs could be clearly identified with Annexin V-FITC, and TRITC-labeled antibodies against CD105 and UPA. As expected, the endothelial MPs remained invisible in case of Ab CD41 (negative control) as can be seen in Supporting Information Figure S7. Sizing of the microparticles was very well possible in plasma (see Figure 5). The broadest size distribution was obtained when using Annexin V that targets phosphatidylserine, which is present on any type of microparticle. The antibody directed against CD105, a membrane glycoprotein constitutively expressed by endothelial cells, detects also a large range of microparticles with a mean size of about $315 \mathrm{~nm}$. However, this marker appeared to be almost absent in small microparticles of around $100 \mathrm{~nm}$, which are preferentially enriched in UPA. UPA is the inducible serine protease expressed by endothelial cells upon stimulation with TNF $\alpha$ 
Endothelial cell-derived microparticles in plasma

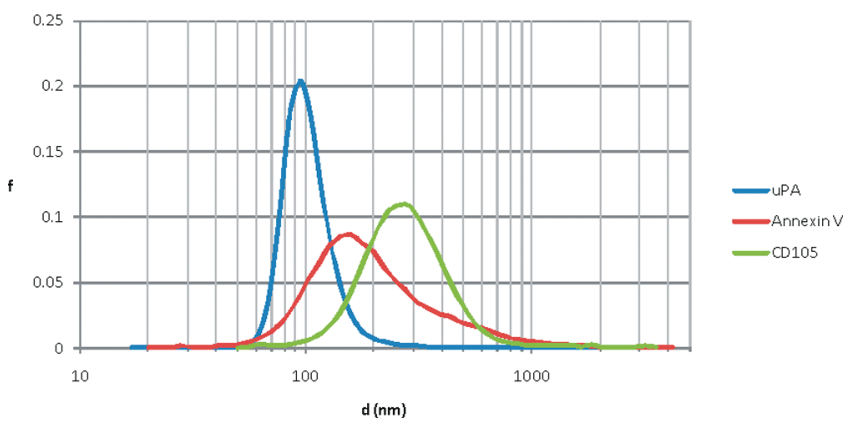

FIGURE 5. Detection and sizing of endothelial-derived microparticles in plasma by fSPT. The endothelial microparticles could be easily detected and sized in plasma after staining with Annexin V-FITC or fluorescently labeled secondary antibodies directed against antibodies to CD105 or UPA (urokinase-type plasminogen activator).

(tumor necrosis factor $\alpha$ ) and seems to be localized in microparticles of a restricted size as shown in Figure 5. These data suggest that different types of microparticles derived from endothelial cells can have a characteristic size range. To the best of our knowledge, this is the first time that cellderived microparticles in the sub-500 $\mathrm{nm}$ range have been identified and characterized in terms of their size in undiluted plasma.

In this study we have given ample evidence that fluorescence SPT in combination with MEM analysis is the first technique suited for real-time accurate sizing of nanoparticles in undiluted complex biological fluids, such as plasma and whole blood. We have shown that MEM analysis significantly improves the precision of the raw size distributions and provides for an improved resolution as compared to DLS size measurements. An additional advantage of fSPT is that only very small sample volumes are required, which can be even less than a microliter. This is beneficial when working with costly materials, as is often the case in the context of drug delivery or when only small amounts of a biological sample are available. Nevertheless, the advantages of fSPT sizing come with some drawbacks as well. While a single fSPT size measurement can be performed in a few seconds, the calculation of the size distribution generally has to be done off-line due to rather intensive image processing calculations for obtaining the trajectories. Also, as for any fluorescence-based technique, care should be taken that the fluorescent labels do not significantly alter the functionality and structure of the nanoparticles being studied. We have verified this explicitly by control experiments using the same particles but with different fluorescent labels (see Supplementary Results in Supporting Information). Furthermore, the particle concentration range for fSPT size measurements is limited due to practical reasons. The upper limit of particle concentration is dictated by the optical resolution and the tracking algorithm, resulting in a maximum useful particle concentration that is typically in the nanomolar range. Recent advancements in tracking algorithms designed for high particle densities might prove to be beneficial in this respect. $^{31,32}$ Theoretically there is no lower limit to the particle concentration as the size is calculated for individual particles. However, it is clear that there should be a sufficient number of particles in the system to obtain good statistics within a reasonable time period.

Until now, sizing nanomatter in whole blood was not possible and diluted serum has been used as a "best" substitute. ${ }^{33,34}$ However, using fSPT we have found that the aggregation of liposomal particles even in undiluted serum and plasma is an underestimate of what happens in blood. This leads to the conclusion that serum and plasma, even when undiluted, can generally not be considered as a reliable substitute for whole blood when one is interested in the size and aggregation of intravenously injected nanomatter. When considering the fSPT sizing data, it seems unlikely that the observed increase in size of liposomes in blood would be caused by platelets or cellular microparticles. Platelets are present in blood at a high concentration of about 200 000/ $\mu \mathrm{L}$, while they are virtually absent in plasma and serum due to centrifugation (and clotting in case of serum). Therefore, if the observed increase in size would be due to adsorption to platelets, then an increase in size would not have been observed in serum and plasma. On the other hand, when considering cell-derived microparticles, it is reported in literature that they are present in low concentrations in blood and plasma from healthy individuals (between 1000 and $3000 / \mu \mathrm{L}$ ). However, the amount of cellular microparticles is much higher in serum since they are abundantly released by platelets during clotting. If the observed increase in size would be due to sticking of the liposomes to cellular microparticles, then aggregation should have been much more pronounced in serum compared to plasma and blood, which was not the case. Therefore, from the fSPT data it seems very unlikely that platelets or cellular microparticles are involved in the aggregation of cationic liposomes. While further research is needed, one possible mediator of liposome aggregation in blood could be fibrinogen, which is more abundantly available in blood than in plasma and serum. Furthermore, in relation to recent findings, it would be interesting to see if the difference between serum and blood might be related to a different composition of the protein corona. ${ }^{5}$ In any case, our results indicate the urgent need for methods that can analyze particle size in undiluted biological media.

When comparing nonpegylated DOTAP-DOPE liposomes with two types of pegylated liposomes, it was found that liposomes containing DSPE-PEG proved to be the least prone to aggregation in whole blood, while the ceramide-PEG functionalized liposomes tended to aggregate more over time. The latter observation was not unexpected as it is known that the ceramide-PEG molecules can gradually diffuse out of the liposomes. Very severe aggregation was observed for the nonpegylated liposomes. When the same liposomes were injected intravenously into rats, we found that the larger particles are removed very rapidly from the 
blood circulation. In agreement with the results from the artificial blood circulation system, this was most evident for the nonpegylated liposomes, and to a lesser extent for the ceramide-PEG and DSPE-PEG DOTAP-DOPE liposomes. The main conclusion from this is that aggregation experiments should at first be carried out in a closed in vitro artificial blood circulation system to assess the extent of aggregation. This can then be followed by in vivo experiments to relate the aggregation of the particles to the clearance from the blood circulation. In future research, we will focus on the development of a suitable algorithm for the calculation of meaningful number concentrations from fSPT measurements. This would further extend the usefulness of the fSPT method to particle clearance experiments and could provide additional information on particle number and size when compared to concentration measurements that rely on the global radioactivity or fluorescence signal of a blood sample. ${ }^{35,36}$

Finally, we have demonstrated that the fSPT sizing technique can be used for identifying and sizing natural cellderived microparticles that were spiked in plasma, provided that specific fluorescent probes (Annexin V, specific antibodies) are used. In further research, we will explore the possibility to label specific types of cellular microparticles directly in pathological plasma. This would be a great benefit as it avoids the elaborate purification steps normally needed to isolate cellular MPs. Therefore, fSPT is expected to contribute to elucidating both the pathophysiological role of cell-derived MPs and their use as disease biomarkers.

In conclusion, we have demonstrated the first study on nanoparticle aggregation in undiluted biological fluids, which was possible by using fSPT combined with MEM analysis. We expect that fSPT will become an important tool in pharmacy, biomedical imaging and diagnostics, as well as other fields where the characterization of nanomatter in complex fluids is of critical importance.

Acknowledgment. The authors thank Annelies Colman and Brecht Moerenhout for their help with initial experiments. The authors are indebted to Dr. Florence Toti for kindly providing Annexin-V FITC. The authors thank Professor Dr. Joris Delanghe for helpful discussions on particle aggregation in blood. Financial support by the Ghent University Special Research Fund and the Fund for Scientific Research Flanders (Belgium) is acknowledged with gratitude. Financial support from FP7 (Arise) is greatly appreciated.

Supporting Information Available. The following information is available: full Materials and Methods, Theory section on maximum entropy analysis of ISPT sizing data, Supplementary Results on the validation of the fSPT sizing technique, Supplementary Figures, and Supplementary Movies. This material is available free of charge via the Internet at http://pubs.acs.org.

\section{REFERENCES AND NOTES}

(1) Nel, A. E.; Madler, L.; Velegol, D.; Xia, T.; Hoek, E. M. V.; Somasundaran, P.; Klaessig, F.; Castranova, V.; Thompson, M.
Understanding biophysicochemical interactions at the nano-bio interface. Nat. Mater. 2009, 8 (7), 543-557.

(2) Nune, S. K.; Gunda, P.; Thallapally, P. K.; Lin, Y. Y.; Forrest, M. L.; Berkland, C. J. Nanoparticles for biomedical imaging. Expert Opin. Drug Delivery 2009, 6 (11), 1175-1194.

(3) Allen, T. M.; Cullis, P. R. Drug delivery systems: Entering the mainstream. Science 2004, 303 (5665), 1818-1822.

(4) Remaut, K.; Sanders, N. N.; De Geest, B. G.; Braeckmans, K.; Demeester, J.; De Smedt, S. C. Nucleic acid delivery: Where material sciences and bio-sciences meet. Mater. Sci. Eng., R 2007, 58 (3-5), 117-161.

(5) Lundqvist, M.; Stigler, J.; Elia, G.; Lynch, I.; Cedervall, T.; Dawson, K. A. Nanoparticle size and surface properties determine the protein corona with possible implications for biological impacts. Proc. Natl. Acad. Sci. U.S.A. 2008, 105 (38), 14265-14270.

(6) Walczyk, D.; Bombelli, F. B.; Monopoli, M. P.; Lynch, I.; Dawson, K. A. What the Cell "Sees" in Bionanoscience. J. Am. Chem. Soc. 2010, 132 (16), 5761-5768.

(7) Decuzzi, P.; Godin, B.; Tanaka, T.; Lee, S. Y.; Chiappini, C.; Liu, $\mathrm{X}$; Ferrari, M. Size and shape effects in the biodistribution of intravascularly injected particles. J. Controlled Release 2010, 141 (3), 320-327.

(8) Gaumet, M.; Vargas, A.; Gurny, R.; Delie, F. Nanoparticles for drug delivery: The need for precision in reporting particle size parameters. Eur. J. Pharm. Biopharm. 2008, 69 (1), 1-9.

(9) Nagayasu, A.; Uchiyama, K.; Kiwada, H. The size of liposomes: a factor which affects their targeting efficiency to tumors and therapeutic activity of liposomal antitumor drugs. Adv. Drug Delivery Rev. 1999, 40 (1-2), 75-87.

(10) Koide, H.; Asai, T.; Hatanaka, K.; Urakami, T.; Ishii, T.; Kenjo, E.; Nishihara, M.; Yokoyama, M.; Ishida, T.; Kiwada, H.; Oku, N. Particle size-dependent triggering of accelerated blood clearance phenomenon. Int. J. Pharm. 2008, 362 (1-2), 197-200.

(11) Rejman, J.; Oberle, V.; Zuhorn, I. S.; Hoekstra, D. Size-dependent internalization of particles via the pathways of clathrin-and caveolae-mediated endocytosis. Biochem. J. 2004, 377, 159-169.

(12) van $\operatorname{der}$ Aa, M. A. E. M.; Huth, U. S.; Hafele, S. Y.; Schubert, R.; Oosting, R. S.; Mastrobattista, E.; Hennink, W. E.; Peschka-Suss, R.; Koning, G. A.; Crommelin, D. J. A. Cellular uptake of cationic polymer-DNA complexes via caveolae plays a pivotal role in gene transfection in COS-7 cells. Pharm. Res. 2007, 24 (8), 1590-1598.

(13) Jiang, J. K.; Oberdorster, G.; Biswas, P. Characterization of size, surface charge, and agglomeration state of nanoparticle dispersions for toxicological studies. J. Nanopart. Res. 2009, 11 (1), 77 89.

(14) Montes-Burgos, I.; Walczyk, D.; Hole, P.; Smith, J.; Lynch, I.; Dawson, K. Characterisation of nanoparticle size and state prior to nanotoxicological studies. J. Nanopart. Res. 2010, 12 (1), 47 53.

(15) van Gaal, E. V.; Spierenburg, G.; Hennink, W. E.; Crommelin, D. J.; Mastrobattista, E. Flow cytometry for rapid size determination and sorting of nucleic acid containing nanoparticles in biological fluids. J. Controlled Release 2010, 141 (3), 328-338.

(16) Bausinger, R.; von Gersdorff, K.; Braeckmans, K.; Ogris, M.; Wagner, E.; Brauchle, C.; Zumbusch, A. The transport of nanosized gene carriers unraveled by live-cell imaging. Angew. Chem., Int. Ed. 2006, 45 (10), 1568-1572.

(17) Levi, V.; Gratton, E. Exploring dynamics in living cells by tracking single particles. Cell Biochem. Biophys. 2007, 48 (1), 1-15.

(18) Saxton, M. J.; Jacobson, K. Single-particle tracking: Applications to membrane dynamics. Annu. Rev. Biophys. Biomol. Struct. 1997, 26, 373-399

(19) Suh, J.; Dawson, M.; Hanes, J. Real-time multiple-particle tracking: applications to drug and gene delivery. Adv. Drug Delivery Rev. 2005, 57 (1), 63-78.

(20) Schaertl, W.; Sillescu, H. Dynamics of Colloidal Hard-Spheres in Thin Aqueous Suspension Layers - Particle Tracking by Digital Image-Processing and Brownian Dynamics Computer-Simulations. J. Colloid Interface Sci. 1993, 155 (2), 313-318.

(21) Allen, C.; Dos Santos, N.; Gallagher, R.; Chiu, G. N. C.; Shu, Y.; Li, W. M.; Johnstone, S. A.; Janoff, A. S.; Mayer, L. D.; Webb, M. S.; Bally, M. B. Controlling the physical behavior and biological performance of liposome formulations through use 
NANO

of surface grafted poly(ethylene glycol). Biosci. Rep. 2002, 22 (2), 225-250

(22) Immordino, M. L.; Dosio, F.; Cattel, L. Stealth liposomes: review of the basic science, rationale, and clinical applications, existing and potential. Int. J. Nanomed. 2006, 1 (3), 297-315.

(23) Webb, M. S.; Saxon, D.; Wong, F. M. P.; Lim, H. J.; Wang, Z.; Bally, M. B.; Choi, L. S. L.; Cullis, P. R.; Mayer, L. D. Comparison of different hydrophobic anchors conjugated to poly(ethylene glycol): Effects on the pharmacokinetics of liposomal vincristine. Biochim. Biophys. Acta 1998, 1372 (2), 272-282.

(24) Shi, F. X.; Wasungu, L.; Nomden, A.; Stuart, M. C. A.; Polushkin, E.; Engberts, J. B. F. N.; Hoekstra, D. Interference of poly(ethylene glycol)-lipid analogues with cationic-lipid-mediated delivery of oligonucleotides; role of lipid exchangeability and non-lamellar transitions. Biochem. J. 2002, 366, 333-341

(25) Akinc, A.; Goldberg, M.; Qin, J.; Dorkin, J. R.; Gamba-Vitalo, C.; Maier, M.; Jayaprakash, K. N.; Jayaraman, M.; Rajeev, K. G.; Manoharan, M.; Koteliansky, V.; Rohl, I.; Leshchiner, E. S.; Langer, R.; Anderson, D. G. Development of Lipidoid-siRNA Formulations for Systemic Delivery to the Liver. Mol. Ther. 2009, 17 (5), 872-879.

(26) Freyssinet, J. M. Cellular microparticles: what are they bad or good for. J. Thromb. Haemostasis 2003, 1 (7), 1655-1662.

(27) Chironi, G. N.; Boulanger, C. M.; Simon, A.; Dignat-George, F.; Freyssinet, J. M.; Tedgui, A. Endothelial microparticles in diseases. Cell Tissue Res. 2009, 335 (1), 143-151.

(28) Doeuvre, L.; Plawinski, L.; Toti, F.; Angles-Cano, E. Cell-derived microparticles: a new challenge in neuroscience. J. Neurochem. 2009, 110 (2), 457-468.
(29) Hugel, B.; Carmen, M.; Martinez, M. C.; Kunzelmann, C.; Freyssinet, J. M. Membrane microparticles: Two sides of the coin. Physiology 2005, 20, 22-27.

(30) Beyer, C.; Pisetsky, D. S. The role of microparticles in the pathogenesis of rheumatic diseases. Nat. Rev. Rheumatol. 2010, $6(1), 21-29$.

(31) Jaqaman, K.; Loerke, D.; Mettlen, M.; Kuwata, H.; Grinstein, S.; Schmid, S. L.; Danuser, G. Robust single-particle tracking in livecell time-lapse sequences. Nat. Methods 2008, 5 (8), 695-702.

(32) Serge, A.; Bertaux, N.; Rigneault, H.; Marguet, D. Dynamic multiple-target tracing to probe spatiotemporal cartography of cell membranes. Nature Methods 2008, 5 (8), 687-694.

(33) Gaber, M. H. Effect of bovine serum on the phase transition temperature of cholesterol-containing liposomes. J. Microencapsulation 1998, 15 (2), 207-214

(34) O’Donnell, R. T.; Martin, S. M.; Ma, Y.; Zamboni, W. C.; Tuscano, J. M. Development and characterization of CD22-targeted pegylated-liposomal doxorubicin (IL-PLD). Invest. New Drugs 2010, 28, 260-297.

(35) Morille, M.; Montier, T.; Legras, P.; Carmoy, N.; Brodin, P.; Pitard, B.; Benoit, J. P.; Passirani, C. Long-circulating DNA lipid nanocapsules as new vector for passive tumor targeting. Biomaterials 2010, 31 (2), 321-329.

(36) Tagami, T.; Nakamura, K.; Shimizu, T.; Ishida, T.; Kiwada, H. Effect of siRNA in PEG-coated siRNA-lipoplex on anti-PEG IgM production. J. Controlled Release 2009, 137 (3-4), 234-240.

(c) 2010 American Chemical Society

4442

DOI: 10.1021/nl103264u | Nano Lett. 2010, 10, 4435--4442 